\title{
Ecocardiografia Tridimensional Revela o Verdadeiro Inimigo em um Jovem de Sexo Masculino com Infarto do Miocárdio com Supradesnivelamento do Segmento ST e Regurgitação Mitral Grave: "Pseudo-Fenda" Posterior e Prolapso da Valva Mitral
}

\author{
Three-dimensional Echocardiography Reveals the True Enemy in a Young Male with ST-Elevation Myocardial \\ Infarction and Severe Mitral Regurgitation: Posterior Mitral Valve "Pseudo-Cleft" and Prolapse
}

\author{
Sorina Mihaila, ${ }^{10}$ Andreea Elena Velcea, ${ }^{1}$ Luigi Paolo Badano, ${ }^{2,3}$ Vinereanu Dragos, ${ }^{1}$ Denisa Muraru ${ }^{2,3}$ \\ University of Medicine and Pharmacy Carol Davila Bucharest - Emergency University Hospital Bucharest, ${ }^{1}$ Bucharest - Romênia \\ Istituto Auxologico Italiano, IRCCS, Department of cardiac, neural and metabolic sciences, San Luca Hospital, ${ }^{2}$ Milão - Itália \\ University of Milano-Bicocca, Department of Medicine and Surgery ${ }^{3}$ Milão - Itália
}

\section{Introdução}

A ecocardiografia tridimensional (E3D) desempenha um papel cada vez mais importante no diagnóstico das valvopatias, na avaliação da morfologia valvar de maneira anatômica e no estabelecimento da reparabilidade da valva, sem ter as limitações da ecocardiografia bidimensional (E2D) convencional. ${ }^{1}$

Nós relatamos o caso de um paciente jovem que apresentou infarto agudo do miocárdio anterior com supradesnivelamento do segmento ST e regurgitação mitral (RM) grave, após intervenção coronária percutânea (ICP) primária bem-sucedida da artéria descendente anterior esquerda, cuja ecocardiografia transesofágica tridimensional (ETE 3D) revelou uma causa inesperada da RM, a saber, patologia complexa da valva mitral (VM) constituindo prolapso dos escalopes P2-3, flail de corda e pseudo-fenda do folheto posterior separando o segmento P1 do segmento P2.

\section{Relado de Caso}

Um paciente do sexo masculino, 38 anos de idade, sem fatores de risco cardiovascular conhecidos, apresentou quadro início agudo de dor torácica constritiva. O exame cardíaco revelou ritmo regular, sopro sistólico apical e pressão arterial normal. O eletrocardiograma de 12 derivações em repouso de emergência mostrou elevação do segmento ST nas derivações $V_{1-6}$ e taquicardia ventricular não sustentada recorrente. A angiografia coronária de emergência mostrou oclusão trombótica aguda da artéria descendente anterior esquerda proximal, estenose não crítica da artéria coronária direita e estenose de 90\% da artéria circunflexa esquerda. Foi realizada ICP primária com implante

\section{Palavras-chave}

Valva Mitral/anormalidades; Infarto do Miocárdio; Ecocardiografia Tridimensional/métodos; Diagnóstico por Imagem; Adulto Jovem.

\section{Correspondência: Sorina Mihaila Baldea •}

Splaiul Independentei no 169, sector 5, 10th floor, Bucharest, Romania -

Cardiology and Cardiovascular Surgery Department University Hospital of

Bucharest, Romania. CEP: 050098

Email: sorinamihaila1981@gmail.com

Artigo recebido em 23/07/2019, revisado em 11/06/2020, aceito em 05/08/2020

DOI: https://doi.org/10.36660/abc.20190485 de stent na artéria descendente anterior esquerda, com bons resultados procedurais.

A E2D transtorácica pós-procedimento mostrou ventrículo esquerdo (VE) não dilatado, anormalidades do movimento da parede septal e discreta disfunção sistólica do VE (fração de ejeção do $\mathrm{VE}=50 \%$ ), bem como dilatação moderada do átrio esquerdo (AE) e RM grave com um jato excêntrico, dirigido anteriormente para o AE (regurgitação holossistólica; área efetiva do orifício regurgitante $=0,4 \mathrm{~cm}^{2}$; volume regurgitante $=55 \mathrm{ml} /$ $\mathrm{m}^{2}$ ). Um discreto prolapso do folheto posterior da VM também foi detectado pela ecocardiografia transtorácica bidimensional (ETT 2D). No entanto, nem a anormalidade do movimento da parede septal nem o prolapso da VM, visto por ETT 2D, explicaram completamente a gravidade da RM. Neste contexto, os mecanismos e a gravidade da RM foram mais explorados por meio da ecocardiografia transesofágica, incluindo avaliação por E3D. A avaliação da VM por ETE 3D da "visão cirúrgica" mostrou prolapso dos segmentos $P_{2-3}$ (Figura 1, Painel A), ruptura de corda fixada no folheto posterior da VM e uma indentação profunda na VM posterior (Figura 1, Painel B), levando a um jato regurgitante excêntrico no $\mathrm{AE}$ até as veias pulmonares. Com a finalidade de determinar a reparabilidade da VM, foi realizado o exame com avaliação da VM por ETE 3D da visão ventricular (Figura 1, Painel C), onde foi detectado uma pseudo-fenda do folheto posterior, com o escalope $P_{1}$ separados dos segmentos de prolapso $P_{2-3}$. A ETE 2D em cores mostrou um jato "dividido" de RM (Figura 2, Painel A), enquanto a ETE 3D em cores mostrou um jato excêntrico de RM, com origem ampla, direcionado anteriormente (Figura 2, Painéis B e C), explicando melhor o mecanismo da RM.

Foram excluídas potenciais causas adquiridas desses achados morfológicos, tais como trauma prévio da VM, cirurgia ou endocardite infecciosa e o diagnóstico final foi de RM grave devido ao prolapso complexo da VM dos segmentos $\mathrm{P}_{2-3}$ e ruptura da corda fixada no folheto posterior da VM, associada a uma pseudo-fenda do folheto posterior entre os segmentos $\mathrm{P}_{1} \mathrm{e}$ $\mathrm{P}_{2}$. O paciente foi encaminhado para parecer cirúrgico, devido ao aumento do AE (mostrando evolução prolongada da RM) e novo aparecimento de sintomas após o evento agudo (dispneia de exercício). Foram realizados com sucesso o reparo da VM, incluindo ressecção do prolapso, sutura da pseudo-fenda da VM e anuloplastia mitral, associados ao enxerto da artéria circunflexa esquerda. No seguimento de três anos, o paciente não apresentou recidiva da $\mathrm{RM}$. 


\section{Relato de Caso}

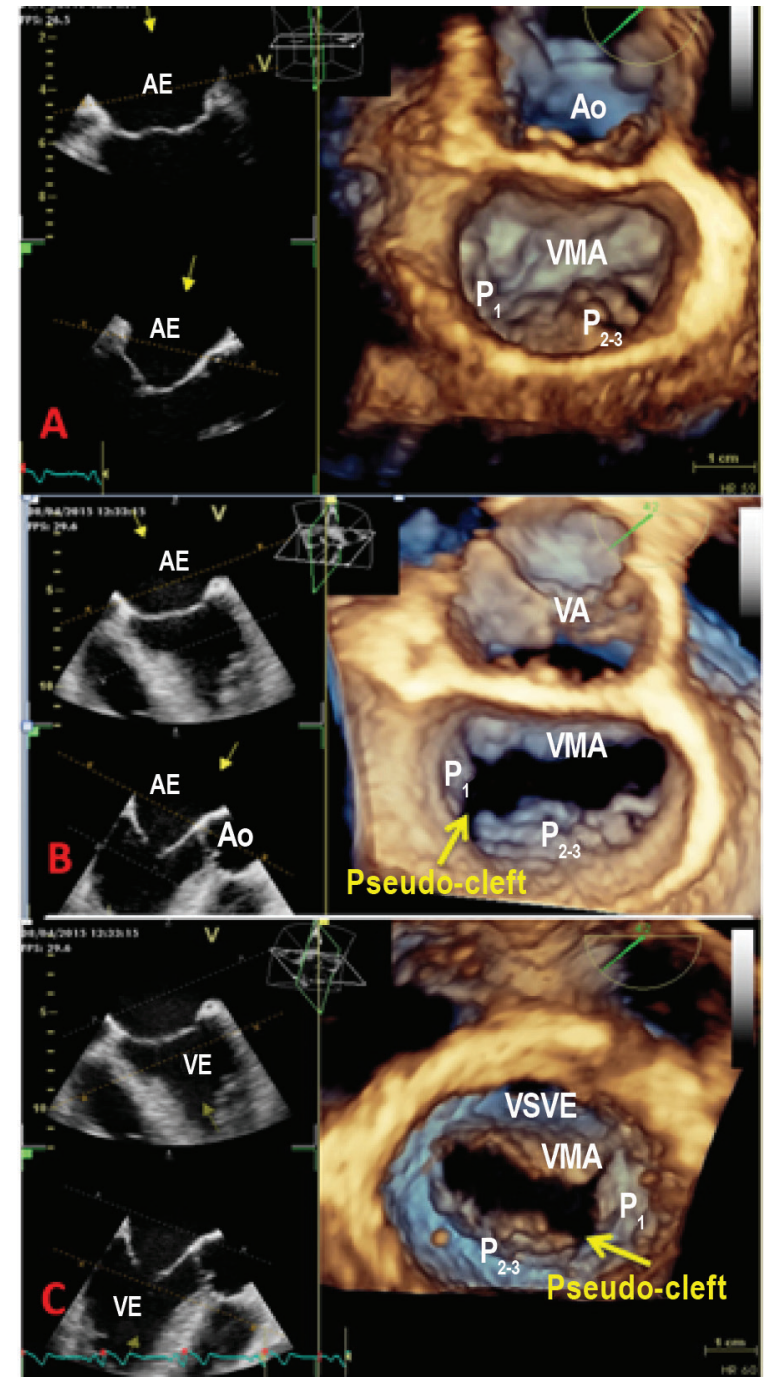

Figura 1 - Avaliação morfológica tridimensional da valva mitral (abordagem transesofágica). Painel A) "Visão cirúrgica" da valva mitral fechada, do lado do átrio esquerdo, que mostra o prolapso complexo dos escalopes $P$. $O$ folheto anterior da valva mitral tem morfologia normal. Painel B) Abêrtura da valva mitral revela que o segmento $P$ está separado dos segmentos $P$, levantando a suspeita de uma pseudo-fenda. Painel C) Valva mitral vistializada do lado do ventrículo esquerdo. A pseudo-fenda do folheto posterior da valva mitral, entre os segmentos $P$ e $P$, pode ser identificada. AE: átrio esquerdo, Ao: aorta, RM: regurgittação3 mitral, VA: valva aórtica, VE: ventrículo esquerdo, VMA: valva mitral anterior, VSVE: via de saída do ventrículo esquerdo.

\section{Discussão}

Nosso caso clínico mostra a utilidade da E3D para o diagnóstico e avaliação morfológica de lesões complexas da VM, principalmente quando a etiologia é incerta, bem como o seu papel no planejamento de procedimentos cirúrgicos. A suspeita inicial da etiologia da RM era isquêmica; entretanto, o curto período de isquemia (menos de 2 horas até a revascularização), as pequenas anormalidades no movimento da parede do VE e a boa função sistólica do VE
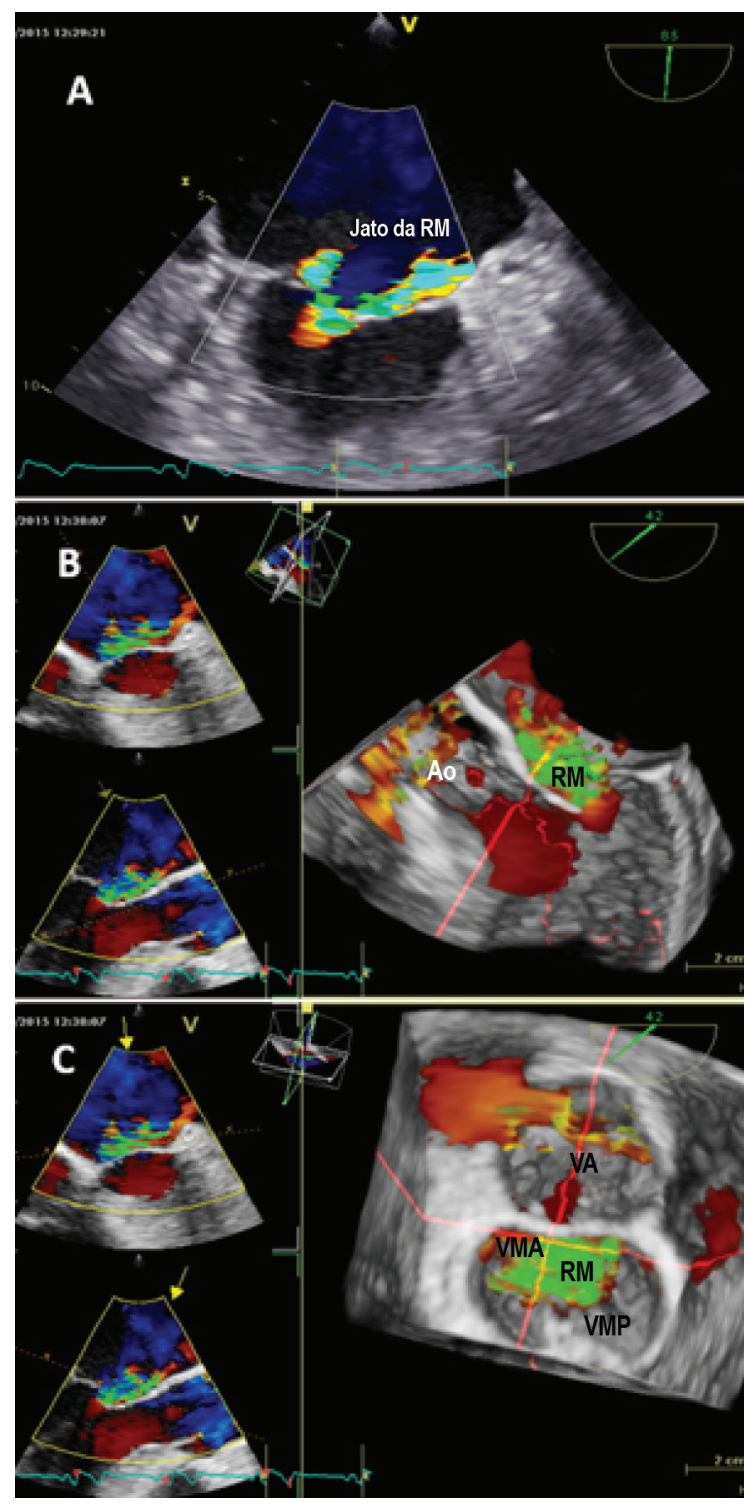

Figura 2 - Avaliação bi- e tridimensional em cores da regurgitação mitral (abordagem transesofágica). Painel A) A valva mitral, visualizada a 85 graus, mostra os dois componentes da regurgitação mitral, causada pelo prolapso $e$ pela pseudo-fenda. Painel B) A visão de eixo longo dos escalopes A2/P2 mostra o jato da regurgitação mitral causado pelo prolapso de $P 2$, oposto ao escalope. Painel C) A "visão cirúrgica" da valva mitral mostra a origem ampla do jato da regurgitação mitral, visto do átrio esquerdo, que é direcionado anteriormente (são exibidos apenas os fluxos retrógrados). Ao: aorta; RM: regurgitação mitral, VA: valva aórtica, VMA: valva mitral anterior, VMP: valva mitral posterior.

tornaram essa causa improvável. A avaliação detalhada da E2D revelou prolapso leve da VM posterior, que também foi insuficiente para explicar a gravidade da RM. Por outro lado, a ETE 3D revelou o prolapso complexo da VM dos segmentos, a ruptura de corda e a pseudo-fenda do folheto posterior da VM separando o escalope $P_{1}$ do $P_{2}$.

Hipotetiza-se que as fendas são resultado da expressão incompleta de um defeito do coxim endocárdico, na maioria 
das vezes envolvendo a parte central do folheto anterior da $\mathrm{VM}^{2,3}$ Fendas verdadeiras que afetam a VM posterior são extremamente raras, ${ }^{2}$ no entanto, as pseudo-fendas são uma classe separada de anomalias morfológicas do folheto posterior da VM. As pseudo-fendas são indentações profundas que compartilham a localização das fendas normais entre os escalopes da VM posterior, mas com mais de $50 \%$ da profundidade dos escalopes adjacentes. ${ }^{4}$ Esta anomalia está frequentemente associada à rotação anti-horária dos músculos papilares, músculo papilar acessório ou folheto da VM acessório, e prolapso da válvula mitral. ${ }^{5}$ Nosso paciente apresentou RM como consequência de prolapso complexo da VM com ruptura de corda, associado à pseudo-fenda. As altas pressões diastólicas finais do VE no contexto do evento isquêmico e a disfunção sistólica do VE provavelmente pioraram a gravidade da RM, pois o paciente negava dispneia antes da internação hospitalar. Além disso, permanece a dúvida se a ruptura da corda ocorreu antes ou se estava relacionada ao evento isquêmico.

No entanto, apesar de não ser totalmente responsável pela $\mathrm{RM}$, a presença da pseudo-fenda tem influência adicional na decisão cirúrgica quanto à reparabilidade da VM. Mantovani et al., ${ }^{6}$ mostraram que $35 \%$ dos pacientes com prolapso da VM apresentavam pseudo-fendas, não vistas pela E2D e reveladas apenas pela E3D. A presença de pseudo-fendas não resolvidas em pacientes com prolapso da VM foi associada a pior prognóstico após o reparo da VM e maior recorrência da RM no seguimento. Neste contexto, foi realizado o reparo da VM em nosso paciente, incluindo a sutura da pseudo-fenda da VM.

\section{Conclusões}

A ETE 3D é uma técnica útil e viável para o diagnóstico correto em pacientes com doença complexa da VM, especialmente quando a etiologia é incerta, bem como para a determinação da a reparabilidade da valva. Embora as pseudo-fendas da VM raramente levem à regurgitação, elas estão associadas a piores desfechos pós-operatórios; portanto, precisam ser suturadas durante o reparo da VM.

\section{Contribuição dos autores}

Concepção e desenho da pesquisa: Baldea SM, Vinereanu D; Obtenção de dados e Redação do manuscrito: Baldea SM, Velcea $A E ;$ Análise e interpretação dos dados: Baldea SM, Badano LP, Muraru D; Revisão crítica do manuscrito quanto ao conteúdo intelectual importante: Baldea $S M$, Velcea $A E$, Badano LP, Vinereanu D, Muraru D.

\section{Potencial conflito de interesses}

Declaro não haver conflito de interesses pertinentes.

\section{Fontes de financiamento}

O presente estudo não teve fontes de financiamento externas.

\section{Vinculação acadêmica}

Não há vinculação deste estudo a programas de pósgraduação.

\section{Aprovação ética e consentimento informado}

Este estudo foi aprovado pelo Comitê de Ética do Emergency University Hospital Bucharest sob o número de protocolo 15/2.07. Todos os procedimentos envolvidos nesse estudo estão de acordo com a Declaração de Helsinki de 1975, atualizada em 2013. O consentimento informado foi obtido de todos os participantes incluídos no estudo

\section{Referências}

1. Lang RM, Mor-Avi V, Sugeng L, Nieman PS, Sahn DJ. Three-dimensional echocardiography: the benefits of the additional dimension. J Am Coll Cardiol. 2006;48(10):2053-69.

2. Amin A, Davis M, Auseon A. Isolated cleft posterior mitral valve leaflet: an uncommon cause of mitral regurgitation. European journal of echocardiography : J Am Soc Echocardiogr. 2009;10(1):173-4.

3. Miglioranza MH, Muraru D, Mihaila S, Haertel JC, Iliceto S, Badano LP. Isolated Anterior Mitral Valve Leaflet Cleft: 3D Transthoracic EchocardiographyGuided Surgical Strategy. Arq Bras Cardiol. 2015;104(5):e49-52.
4. Narang A, Addetia K, Weinert L, Yamat M, Shah AP, Blair JE, et al. Diagnosis of Isolated Cleft Mitral Valve Using Three-Dimensional Echocardiography. J Am Soc Echocardiogr. 2018;31(11):1161-7.

5. McEnany MT, English TA, Ross DN. The congenitally cleft posterior mitral valve leaflet. An anticedent to mitral regurgitation. Ann Thorac Surg. 1973;16(3):281-92

6. Mantovani F, Clavel MA, Vatury O, Suri RM, Mankad SV, Malouf J, et al Cleft-like indentations in myxomatous mitral valves by three-dimensional echocardiographic imaging. Heart. 2015;101(14):1111-7. 